\title{
Electroresponsive Nanoparticles Improve Antiseizure Effect of Phenytoin in Generalized Tonic-Clonic Seizures
}

\author{
Yi Wang ${ }^{1} \cdot$ Xiaoying Ying ${ }^{1,2} \cdot$ Liying Chen ${ }^{1} \cdot$ Yao Liu ${ }^{1}$ - Ying Wang ${ }^{1} \cdot$ Jiao Liang $^{1}$ •

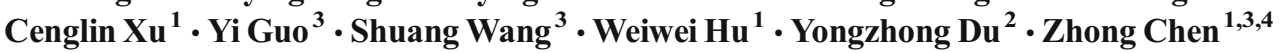

Published online: 2 May 2016

(C) The American Society for Experimental NeuroTherapeutics, Inc. 2016
In both electrical- (maximal electrical shock) and chemicalinduced (pentylenetetrazole and pilocarpine) seizure models, ANG-PHT-ERHNPs lowered the effective therapeutic doses of PHT and demonstrated the improved antiseizure effects compared with ANG-PHT-HNPs or PHT solution. These results demonstrate that ANG-ERHNPs are able to transport PHT into the brain efficiently and release them when epileptiform activity occurred, which is due to the content of sodium 4-vinylbenzene sulfonate in formula. This may change the therapeutic paradigm of existing drug treatment for epilepsy into a type of on-demand control for epilepsy in the future.

Key Words Electroresponsive · Nanoparticle · Sodium 4-vinylbenzene sulfonate $\cdot$ Epilepsy $\cdot$ Microdialysis

\section{Introduction}

Epilepsy is a disease characterized by recurrent seizures, which are transient symptoms of abnormal, excessive, or synchronous neuronal activity in the brain [1]. Antiepileptic drugs (AEDs) are the mainstay of the management of epilepsy for most patients [2]. However, the relatively narrow therapeutic window of AEDs, such as that for phenytoin sodium (PHT), result in serious potential side effects (metabolic bone disease, peripheral neuropathy, and immunologic effects) [3]. The onset of seizures is often an abrupt, unpredicted event, and AEDs are currently used for preventive treatment of seizure onset, but are not used for on-demand treatment. This situation further contributes to long-term intolerable side effects and inability to control the seizures completely [4]. Thus, safe and effective therapy for epilepsy would ideally require a drug delivery system that can penetrate the blood-brain barrier (BBB) and subsequently release AEDs rapidly to suppress neuronal discharges in a timely manner. 
In the last few years, although numerous drug delivery carriers that can transport AEDs to the brain have been developed, including polymeric nanoparticles [5], polymeric micelles [6], and dendrimers [7], few of these carriers have advanced based on the pathological characteristics of epileptic seizures. We previously designed electroresponsive hydrogel nanoparticles (ERHNP) based on the abnormal electrical activity characteristics of epileptic seizures, and further modified ERHNP with the brain-targeting angiopep-2 peptide (ANG), a ligand of the low-density lipoprotein receptor-related protein, to improve penetration of the BBB [8]. However, the optimal preparation formula for electroresponsive ability is still unclear and the in vivo electroresponsive characteristics of ANG-PHT-ERHNP during seizures has been limited by the challenges of performing molecular studies in the face of generalized convulsions. Although the improved antiepileptic effect of ANG-PHT-ERHNP compared with the PHT solution was demonstrated in the kindling model [8], it is highly likely that the improved antiepileptic effect may be caused by a higher concentration of PHT in the central nervous system (CNS) owing to the brain-targeting effect of ANG. Moreover, PHT is highly bound to proteins both in plasma and in the brain, so only the free (nonprotein-bound) levels of PHT are relevant for its pharmacological effects [9]. There is currently a lack of evidence to address whether or not the free levels of PHT are released from the complex when seizures occur. Thus, in the present study, we aimed to further investigate the in vivo electroresponsive ability of nanoparticles with different formulae by microdialysis and the antiepileptic effects of nanoparticles with experiments conducted with in vivo seizures models.

\section{Methods}

\section{Animals}

Male Sprague-Dawley rats (260-300 g, Grade II, Certificate No. SCXK2008-0033; Experimental Animal Center, Zhejiang Academy of Medical Science, Hangzhou, China) were maintained in cages (12-h light-dark cycle, lights on from 08:00 to 20:00). The rats were housed in groups, with free access to water and food. Experiments were carried out each day between 9:00 and 17:00. All experiments were approved by the Zhejiang University Animal Experimentation Committee, and were performed in complete compliance with the US National Institutes of Health Guide for the Care and Use of Laboratory Animals.

\section{Reagents}

2-Dimethylamino ethyl methacrylate (DMAEMA) and sodium 4-vinylbenzene sulfonate (NaSS) were obtained from
Sigma-Aldrich (St. Louis, MO, USA). $N, N^{\prime}$-Methylene bisacrylamide was purchased from Bio Basic (Markham, ON, Canada). Acrylate-poly (ethylene glycol)- $N$ hydroxysuccinimadyl-ester $(3.5 \mathrm{KDa})$ was obtained from JenKem Technology Co., Ltd. (Beijing, China). Styrene was obtained from Shanghai Lingfeng Reagent Co., Ltd. (Shanghai, China). Potassium persulfate and sodium pyrosulfite were purchased from Sigma-Aldrich. ANG (TFFYGGSRGKRNNFKTEEY, 2.4KDa) was synthesized by Chinese Peptide Company (Hangzhou, China). PHT was purchased from Wuhan Yuancheng Gongchuang Technology Co., Ltd. (Wuhan, China).

\section{Synthesis of ANG-PHT-HNP and ANG-PHT-ERHNP}

In this paper, ERHNPs or HNPs were synthesized using soap-free emulsion copolymerization [10]. Briefly, monomers (Table 1), such as 2-dimethylamino ethyl methacrylate, styrene, or NaSS, were added to $85 \mathrm{ml}$ water in a 250-ml 4-neck round-bottom flask that was equipped with a stirrer, a condenser (nitrogen outlet), a nitrogen inlet tube, and an inlet for the ingredients. Potassium persulfate $(0.1 \mathrm{~g})$ and sodium pyrosulfite $(0.08 \mathrm{~g})$, which were dissolved in $5 \mathrm{ml}$ water, were used as oxidationreduction initiators. A slow stream of nitrogen was introduced into the monomer mixture and the initiator solution for $1 \mathrm{~h}$. The polymerization reaction was conducted with shaking at $160 \mathrm{rpm}$ at $25^{\circ} \mathrm{C}$. After $24 \mathrm{~h}$, the reaction mixture was dialyzed using a dialysis membrane (MWCO 14 kDa; Spectrum Laboratories, Rancho Dominguez, CA, USA) in de-ionized water for $48 \mathrm{~h}$ and was then lyophilized.

ANG-ERHNP or ANG-HNP were further synthesized by coupling the $N$-hydroxysuccinimadyl-ester of the acrylate-poly (ethylene glycol)- $N$-hydroxysuccinimadylester on the surface of the ERHNP or HNP to the $\mathrm{NH}_{2}$ group of ANG. In this reaction, amide crosslinks were formed between $\mathrm{N}$-hydroxysuccinimadyl-ester and $\mathrm{NH}_{2}$. Briefly, $1 \mathrm{ml}$ ANG aqueous solution $(1 \mathrm{mg} / \mathrm{ml})$ was added to $10 \mathrm{ml}$ ERHNP or HNP solution $(10 \mathrm{mg} / \mathrm{ml})$ with a molar ratio of $A N G: P E G$ at 1:1. The reaction solution was stirred overnight at room temperature and then dialyzed against deionized water using a dialysis membrane (MWCO, $14 \mathrm{kDa}$, Spectrum Laboratories) for $24 \mathrm{~h}$ to remove unreacted ANG. The ANG-ERHNP or ANG-HNP were then lyophilized for $24 \mathrm{~h}$.

ANG-PHT-ERHNP or ANG-PHT-HNP was synthesized as follows: $500 \mathrm{mg}$ ANG-ERHNP or ANG-HNP were soaked in $50 \mathrm{ml}$ of $10 \mathrm{mg} / \mathrm{ml}$ PHT solution and collected by centrifugation at $64,400 \mathrm{~g}$ for $60 \mathrm{~min}$ and then lyophilized. The vehicle of PHT solution was alkalized saline $(\mathrm{pH} \sim 8)$ with $0.1 \mathrm{~N}$ $\mathrm{NaOH}$ solution. Please provide the centrifugation as a $\mathrm{g}$ value. $4 \mathrm{mg} / \mathrm{ml}$ 
Table 1 Electroresponsive hydrogen nanoparticles prepared with different formulas

\begin{tabular}{llllllll}
\hline Formula & $\begin{array}{l}\text { DMAEMA } \\
(\%)\end{array}$ & $\begin{array}{l}\text { ST } \\
(\%)\end{array}$ & $\begin{array}{l}\text { NaSS } \\
(\%)\end{array}$ & $\begin{array}{l}\text { MBA } \\
(\%)\end{array}$ & $\begin{array}{l}\text { Yield } \\
(\%)\end{array}$ & $\begin{array}{l}\text { Diameter } \\
(\mathrm{nm})\end{array}$ & $\begin{array}{l}\text { Diameter under electrical field } \\
(\mathrm{nm})\end{array}$ \\
\hline 1 & 93 & 5 & - & 2 & 53.5 & $77.3 \pm 9.0$ & $79.3 \pm 12.0$ \\
2 & 93 & 4 & 1 & 2 & 58.0 & $146.1 \pm 6.2$ & $246.2 \pm 14.7 * *$ \\
3 & 92 & 4 & 2 & 2 & 66.0 & $90.8 \pm 8.9$ & $209.0 \pm 21.0 * *$ \\
4 & 93 & 3 & 2 & 2 & 65.3 & $77.1 \pm 7.0$ & $143.0 \pm 10.8 * *$ \\
5 & 91 & 4 & 3 & 2 & 66.8 & $54.6 \pm 3.4$ & $135.0 \pm 13.5 * *$ \\
\hline
\end{tabular}

DMAEMA = 2-Dimethylamino ethyl methacrylate; $\mathrm{ST}$ = styrene; NaSS = sodium 4-vinylbenzene sulfonate; MBA $=N, N^{\prime}$-methylene bisacrylamide

$* * p<0.01$

\section{Characterization of ANG-PHT-ERHNPs or ANG-PHT-HNPs}

The particle size was determined using a dynamic lightscattering technique with a Zetasizer instrument (Nano S-90; Malvern Instruments, Malvern, UK). To evaluate the effects of an electric field on particle size, $2 \mathrm{ml}$ ANG-ERHNP or ANGHNP $(1 \mathrm{mg} / \mathrm{ml})$ were placed in a glass tube with 2 parallel platinum electrodes ( $1 \mathrm{~cm}$ apart). DC voltage $(50,100,200$, and $500 \mu \mathrm{A}$ current) was then applied to the solution for 1,5 , 10,15 , and $30 \mathrm{~min}$.

For the in vitro release tests, $2 \mathrm{ml}$ ANG-PHT-ERHNP or ANG-PHT-HNP solution $(1 \mathrm{mg} / \mathrm{ml})$ was added into a dialysis bag (MWCO; 8000-14,000) and incubated in $50 \mathrm{ml}$ phosphate-buffered saline (pH 7.4) at $37{ }^{\circ} \mathrm{C}$ with $60 \mathrm{rpm}$ stirring. At the predetermined time points, $1 \mathrm{ml}$ release medium was withdrawn and the PHT concentrations were measured by high-performance liquid chromatography (HPLC). To investigate the release of ANG-PHT-ERHNP or ANG-PHT-HNP solution under electrical field, DC voltage was applied to the solution with current of $100 \mu \mathrm{A}$.

\section{Pharmacokinetic Studies}

Rats were randomly divided into 3 groups and injected (50 mg/kg, i.p.) with PHT solution, ANG-PHT-HNP, or ANG-PHT-ERHNP. Blood samples $(0.5 \mathrm{ml})$ were withdrawn at predetermined time points into heparinized microcentrifuge tubes. The plasma was separated by centrifuging the blood samples at $4000 \mathrm{rpm}$ for $15 \mathrm{~min}$, and the plasma samples were stored at $-20{ }^{\circ} \mathrm{C}$ until analysis.

For the analysis of the PHT concentrations, a 50- $\mu$ sample of plasma was thoroughly mixed with $100 \mu \mathrm{l}$ acetonitrile using a vortexer and centrifuged at $4000 \mathrm{rpm}$ for $10 \mathrm{~min}$. Then, $20 \mu \mathrm{l}$ supernatant was injected for HPLC analysis. The calibration curve we generated for use in the quantification of PHT in the plasma was linear within the concentration range of $0.5-10 \mu \mathrm{g} / \mathrm{ml}$ $(\mathrm{A}=45.61 \mathrm{C}+4.40, r=0.9994)$. The pharmacokinetic parameters were calculated using DAS Version 2.1.1.

\section{Maximal Electroshock-induced Seizures}

A maximal electroshock (MES; $0.2 \mathrm{~s}$ at $50 \mathrm{~Hz}, 150 \mathrm{~mA}$ ) was delivered through ear clips using a rodent shocker (Hugo Sachs Elektronik, March-Hugstetten, Germany); this produced a generalized tonic-clonic seizure, as described in our previous report [11]. Seizure severity was scored by a trained observer who was unaware of experimental groupings. The assessment was based on the extent of the spread of tonic extension and was scored as follows: 1 , clonic seizures, absence of forelimb extension; 2 , tonic seizures accompanying complete forelimb extension without hindlimb extension; 3 , tonic seizures accompanying complete fore- and hindlimb extension. The duration of tonic extension and incidence of tonic seizures were also analyzed as measures of seizure severity. The MES test was performed $1 \mathrm{~h}$ after injection (i.p.) of test drugs between 9:00 and 12:00.

\section{Pentylenetetrazole-induced Seizure}

Under sodium pentobarbital anesthesia (45 mg/kg, i.p.), rats were mounted on a stereotaxic apparatus (512600; Stoelting, Wood Dale, IL, USA). Electrodes were implanted into the right ventral hippocampus (AP: $-5.3 \mathrm{~mm}, \mathrm{~L}:-5.0 \mathrm{~mm}$, V: $5.0 \mathrm{~mm}$ ) for electroencephalography (EEG) recording according to the atlas of rats [12], as per our previously reported protocol $[13,14]$. Two screws were placed in the skull, over the cerebellum, to serve as the reference and ground electrodes. The electrodes were connected to a miniature receptacle that was attached to the skull with dental cement.

After 7 days of recovery, the rats were injected with pentylenetetrazole (PTZ; $80 \mathrm{mg} / \mathrm{kg}$, i.p.), and EEGs were recorded and analyzed using a Neuroscan system (Compumedics, Abbotsford, VIC, Australia), as we reported previously [15, 16]. Seizure severity was classified according to the modified Racine scale [17]: 1 , facial movement; 2 , head nodding; 3 , unilateral forelimb clonus; 4 , bilateral forelimb clonus and rearing; 5 , bilateral forelimb clonus with loss of posture; 6 , generalized tonic-clonic seizures, status epilepticus, or death. Stages $4-6$ as 
generalized seizures (GS) [18]. Rats were observed for a period of 45 min post-PTZ delivery. A trained observer, who was unaware of experimental groupings, scored seizure severity. The test drugs were injected (i.p.) $1 \mathrm{~h}$ prior to PTZ delivery. The PTZ test was performed between 13:00 and 17:00.

\section{Pilocarpine-induced Seizures}

Seizures were induced by intraperitoneal administration of pilocarpine $(30 \mathrm{mg} / \mathrm{kg}) 20 \mathrm{~h}$ after pretreatment with lithium ( $3 \mathrm{mEq} / \mathrm{kg}$, i.p.). Then, rats were observed for a period of $60 \mathrm{~min}$ postpilocarpine delivery. The seizure severity was classified according to a modified Racine scale as mentioned above. A trained observer, who was unaware of experimental groupings, scored seizure severity. The test drugs were injected (i.p.) $1 \mathrm{~h}$ prior to pilocarpine delivery. The pilocarpine test was performed between 13:00 and 17:00.

\section{Microdialysis}

Under sodium pentobarbital anesthesia (45 mg/kg, i.p.), rats were chronically implanted with guide cannulae (CMA/12 polyurethane; Carnegie Medicine, Stockholm, Sweden) into the right ventral hippocampus. The cannula was held in place with 3 screws that were attached to the skull with dental cement.

After 7 days of recovery, microdialysis probes (CMA/12, 3-mm polycarbonate membrane, cut-off $20 \mathrm{kDa}$; Carnegie Medicine) were lowered through the guide cannula to a depth of $5.0 \mathrm{~mm}$ according to the bregma, and the rat was placed in a freely moving system, consisting of a plastic cylinder with a counter-balancing arm carrying a 2-channel swivel (Carnegie Medicine). After balancing for $2 \mathrm{~h}$ after insertion, perfusion of the probe was started using artificial cerebrospinal fluid $(\mathrm{NaCl}$ $125 \mathrm{mM}, \mathrm{KCl} 2.5 \mathrm{mM}, \mathrm{MgCl}_{2} 1.18 \mathrm{mM}$, and $\mathrm{CaCl}_{2}$ $1.26 \mathrm{mM}$ ). The inflow to the probe was driven by a CMA/ 100 microinjection pump (flow rate was $2.0 \mu \mathrm{l} / \mathrm{min}$ ), and a $60 \mu \mathrm{l}$ outflow sample was collected every $30 \mathrm{~min}$. The 2 baseline dialysate samples were collected over a period of $1 \mathrm{~h}$ before the rats were injected with test drugs $(20 \mathrm{mg} / \mathrm{kg}$, i.p.). Following drug administration, a further 8 samples were collected over the next $4 \mathrm{~h}$.

Prior to each microdialysis probe used for the in vivo experiments, in vitro recovery was determined to calculate corrected dialysate concentrations by dialysis of a stirred artificial cerebrospinal fluid solution containing $5 \mu \mathrm{g} / \mathrm{ml}$ phenytoin. The recovery values of individual probes ranged between 21 and $43 \%$.

PHT concentrations in dialysate were analyzed by HPLC with ultraviolet detection. Briefly, a mixture of methanol and water $(55: 45)$ was used as the mobile phase. The flow rate was kept constant at $1.0 \mathrm{ml} / \mathrm{min}$ and the temperature was maintained at $25{ }^{\circ} \mathrm{C}$. Sample aliquots $(20 \mu \mathrm{l})$ were injected for
HPLC analysis using an Agilent 1100 system with a C18 column (Diamonsil, $200 \times 4.6,5 \mu \mathrm{m}$; Dikma Inc., Lake Forest, CA, USA) and ultraviolet detection at $210 \mathrm{~nm}$. All concentrations were calculated from a standard curve of PHT $(0.25-10.0 \mu \mathrm{g} / \mathrm{ml})$ obtained from spiked plasma samples.

\section{Statistics}

Data are presented as the mean \pm SEM. Statistical comparisons were performed using SPSS (version 17.0; IBM, Armonk, NY, USA) with appropriate inferential methods as indicated in the figure legends. $p$-Values $<0.05$ were considered to be statistically significant.

\section{Results}

\section{The In vitro Electroresponsive Behavior of the Nanoparticles with Different Formulae}

We compared 5 different formulae (Table 1), and found that the diameter of formulae $2-5$, but not that of formula 1 , enabled response to an external electric field, suggesting that the presence of NaSS in the hydrogel nanoparticles is a key factor influencing electroresponsive characteristics. Further, we found that the electroresponsive ability of nanoparticle is correlated with the NaSS concentration. The higher concentration of NaSS, the larger fold increased in the diameter of nanoparticles under electrical field (Fig. 1A). However, the content of NaSS is negatively correlated with the original particle size (Fig. 1A).

ANG-ERHNP (formula 3) and ANG-HNP (formula 1) were further prepared for later experiments as electroresponsive nanoparticles and nonelectroresponsive nanoparticles, respectively. Under an external electric field, the particle size of the ANG-HNP remained stable, while the particle size of the ANG-ERHNP increased dramatically as the current increased from 50 to $500 \mu \mathrm{A}$ (Fig. 1B), results consistent with our previous study [8]. The increase in diameter happened as quickly as within $1 \mathrm{~min}$ of exposure to the electric field (Fig. 1C). The in vitro release experiment showed that concentration of accumulated PHT released from the ANG-PHT-ERHNP increased greatly (by nearly $100 \%$ ) under a $100-\mu$ A current (Fig. 1D).

\section{The In vivo Antiepileptic Effect of the Nanoparticles in Epilepsy Models}

In our experiments with the MES model (Fig. 2A), which is commonly used for quickly predicting the clinical anticonvulsant activity of experimental drugs [19], the PHT solution, the ANG-PHT-HNP, and ANG-PHT-ERHNP all relieved the 
a
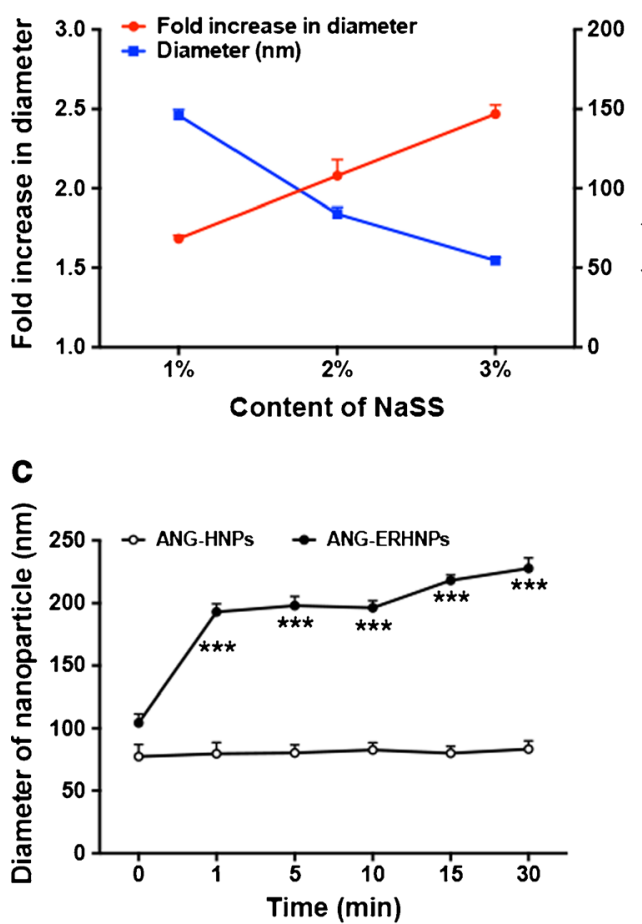

Fig. 1 The in vitro electroresponsive behavior of the nanoparticles with regard to diameter and drug release. (a) The effect of sodium 4vinylbenzene sulfonate (NaSS) content on the original particle size and electroresponsive behavior, which was measured as fold increase in diameter of nanoparticles under a $100-\mu \mathrm{A}$ electric field $(n=3)$; (b) the diameter of the angiopep-2 hydrogel nanoparticles (ANG-HNPs) and ANG electroresponsive HNP (ANG-ERHNP) after the application of

MES-induced seizures in a dose-dependent manner, as compared with the control group. These treatments all lowered the seizure stage and incidence of tonic seizures and shortened tonic seizure duration (Fig. 2B-D). At the dose of $20 \mathrm{mg} / \mathrm{kg}$, the ANG-PHT-ERHNP had a more powerful antiepileptic effect than did the ANG-PHT-HNP (Fig. 2C, D), indicating that ANG-PHT-ERHNP can be administered at lower the effective therapeutic doses.

To exclude the possibility that the released PHT from ANG-PHT-ERHNP was caused by electrical shock but not from the seizure itself, we performed experiments with 2 additional chemical-induced seizure models, which have been shown to be responsive to the PHT tested here $[20,21]$. In the PTZ model (Fig. 3A), at a dose of 10 or $20 \mathrm{mg} / \mathrm{kg}$, the ANG-PHT-ERHNP significantly alleviated the severity of seizures, lowered the incidence of GS, and increased the latency of GS (Fig. 3B-E). It showed a superior antiepileptic effect compared with the PHT solution and the ANG-PHT-HNP, both of which only weakly lowered the incidence of GS at the dose of $20 \mathrm{mg} / \mathrm{kg}$ (Fig. 3C). However, the ANG-PHTERHNP did not lengthen the latency of nonconvulsive seizures (Fig. 3E). Representative epileptic EEGs during seizures and their corresponding energy spectra are shown in Fig. 4A.

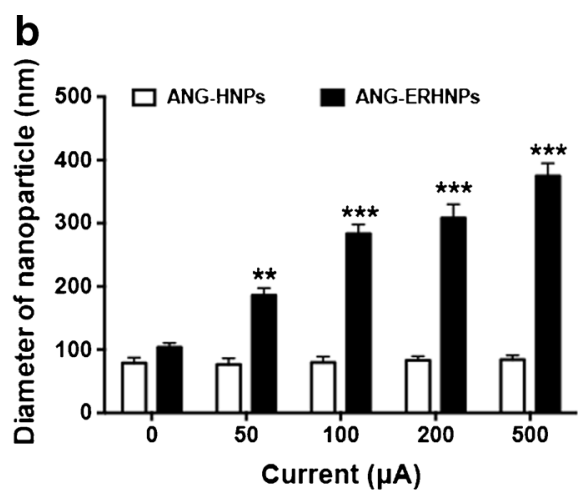

d

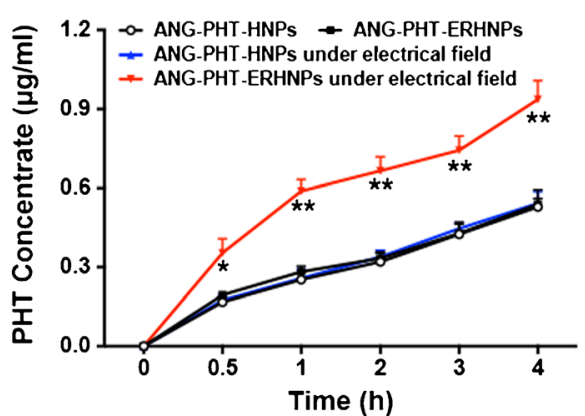

different currents for $10 \min (* * p<0.01, * * * p<0.001, t$-test); (c) the diameter of the ANG-HNPs and ANG-ERHNPs after the application of $100 \mu \mathrm{A}$ current for different times $(* * * p<0.001, t$-test); (d) phenytoin sodium (PHT) released from ANG-PHT-HNPs and ANG-PHT-ERHNPs with or without an external $100-\mu \mathrm{A}$ electric field for $4 \mathrm{~h}\left({ }^{*} p<0.05\right.$, $* * p<0.01)$ compared with first group, 1-way ANOVA with post-hoc fishers least significant difference (LSD) test

The EEG spectrum analysis further showed that ANG-PHTERHNP treatment reduced the severity of the EEG onset at the dose of $20 \mathrm{mg} / \mathrm{kg}$ (Fig. 4B). There were no differences in antiepileptic effect among all groups at the higher dose $(50 \mathrm{mg} / \mathrm{kg})$, probably because of a ceiling effect in these models (Supplementary Fig. S1).

Similarly, in the pilocarpine model (Fig. 5A), the ANGPHT-ERHNP also showed a superior antiepileptic effect compared with the PHT solution and the ANG-PHT-HNP at a dose of 10 or $20 \mathrm{mg} / \mathrm{kg}$ (Fig. 5B-E). Although ANG-PHT-ERHNP did not alleviate the severity of seizures, it significantly lowered the incidence of death (Fig. 5C), and increased the latency of GS (Fig. 5D) and SE (Fig. 5E).

\section{The In vivo Electroresponsive Release of PTH from Nanoparticles During Seizures}

To evaluate directly the seizure-triggered PHT release, we used microdialysis to measure the free level of PHT during seizures (Fig. 6A) [22]. As shown in Fig. 6B, the PHT concentrations of PHT solution plateaued at $60 \mathrm{~min}$ and then began to decrease exponentially after $90 \mathrm{~min}$ in naïve mice. Both ANG-PHT-HNP and ANG-PHT-ERHNP showed a 
Fig. 2 The in vivo antiepileptic effect of the nanoparticles in the maximal electroshock (MES) model. (a) Design of experiment and seizure scores in the MES model; (b-d) the effect of nanoparticles at different doses on seizures stage (b), incidence of tonic seizures (c), and tonic seizure duration (d); the number of rats used in the experiment is indicated in column $\mathrm{B}\left({ }^{*} p<0.05\right.$, ${ }^{* *} p<0.01,{ }^{* * *} p<0.001$ compared with the first group, ${ }^{\#} p<0.05$ compared with groups with the same dose; $\chi^{2}$ tests were used to compare the incidence of tonic seizures; Kruskal-Wallis ANOVA followed by post-hoc Dunn's tests were used to compare seizure stage, and 1-way ANOVA followed by post-hoc fishers least significant difference (LSD) tests were used to compare tonic seizure duration) $\mathrm{PHT}=$ phenytoin sodium; $\mathrm{ANG}=$ angiopep-2; HNP = hydrogel nanoparticles; ERHNP = electroresponsive HNP

Fig. 3 The in vivo antiepileptic effect of the nanoparticles in the pentylenetetrazole (PTZ) model. (a) Design of experiment; (b-e) the effect of nanoparticles at different doses on seizures stage (b), incidence of generalized seizures (GS) (c), latency to GS (d), and latency to nonconvulsive seizure (e). The number of rats used in the experiment is indicated in the column of B $\left({ }^{*} p<0.05,{ }^{* *} p<0.01\right.$,

$* * * p<0.001$ compared with the first group; ${ }^{\#} p<0.05$ compared with groups with same dose; the $\chi^{2}$ tests were used to compare the incidence of GS, Kruskal-Wallis ANOVA followed by post-hoc Dunn's tests were used to compare seizure stage; and 1-way ANOVA followed by a post-hoc fishers least significant difference (LSD) test were used to compare latency to GS and nonconvulsive seizure $\mathrm{PHT}=$ phenytoin sodium; ANG $=$ angiopep-2; HNP = hydrogel nanoparticles; ERHNP = electroresponsive HNP; EEG = electroencephalography
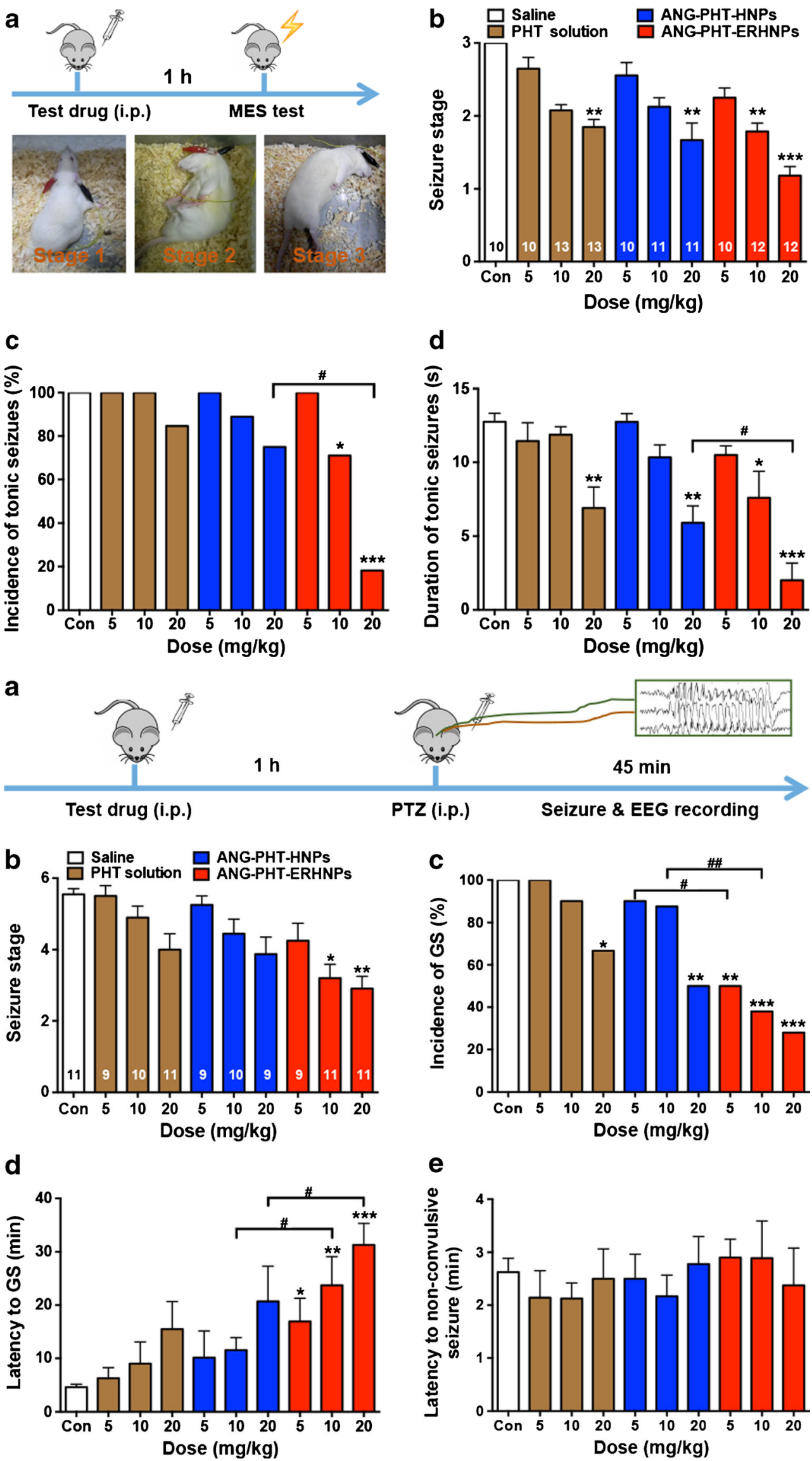
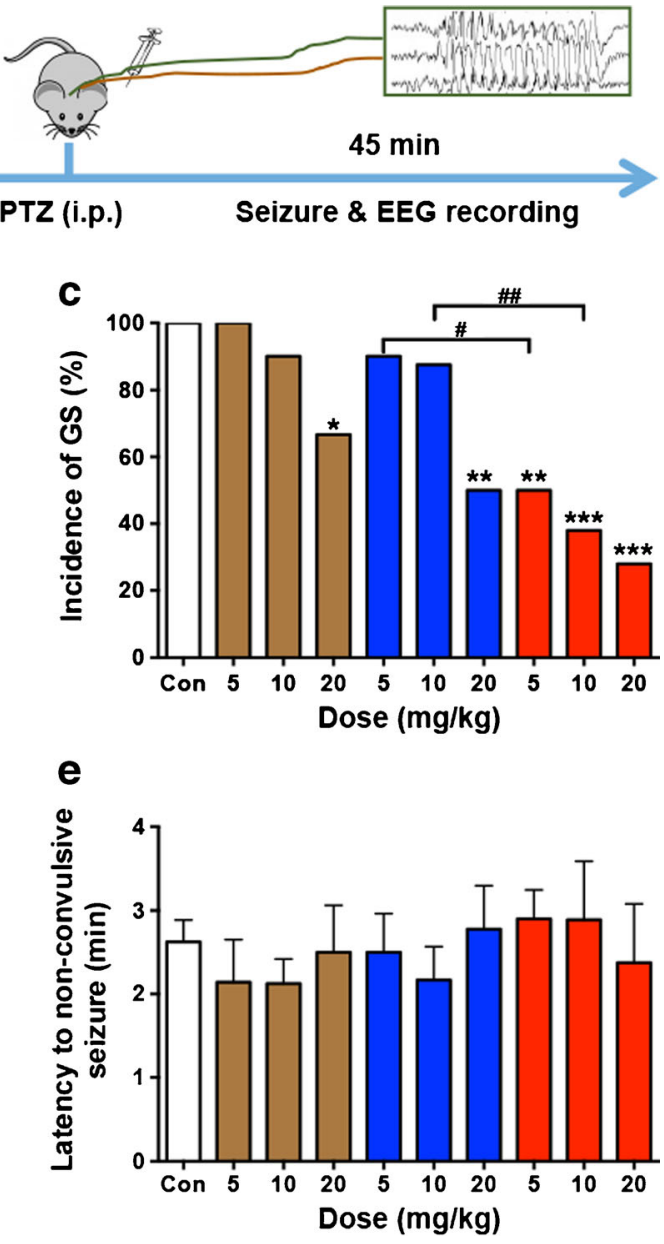

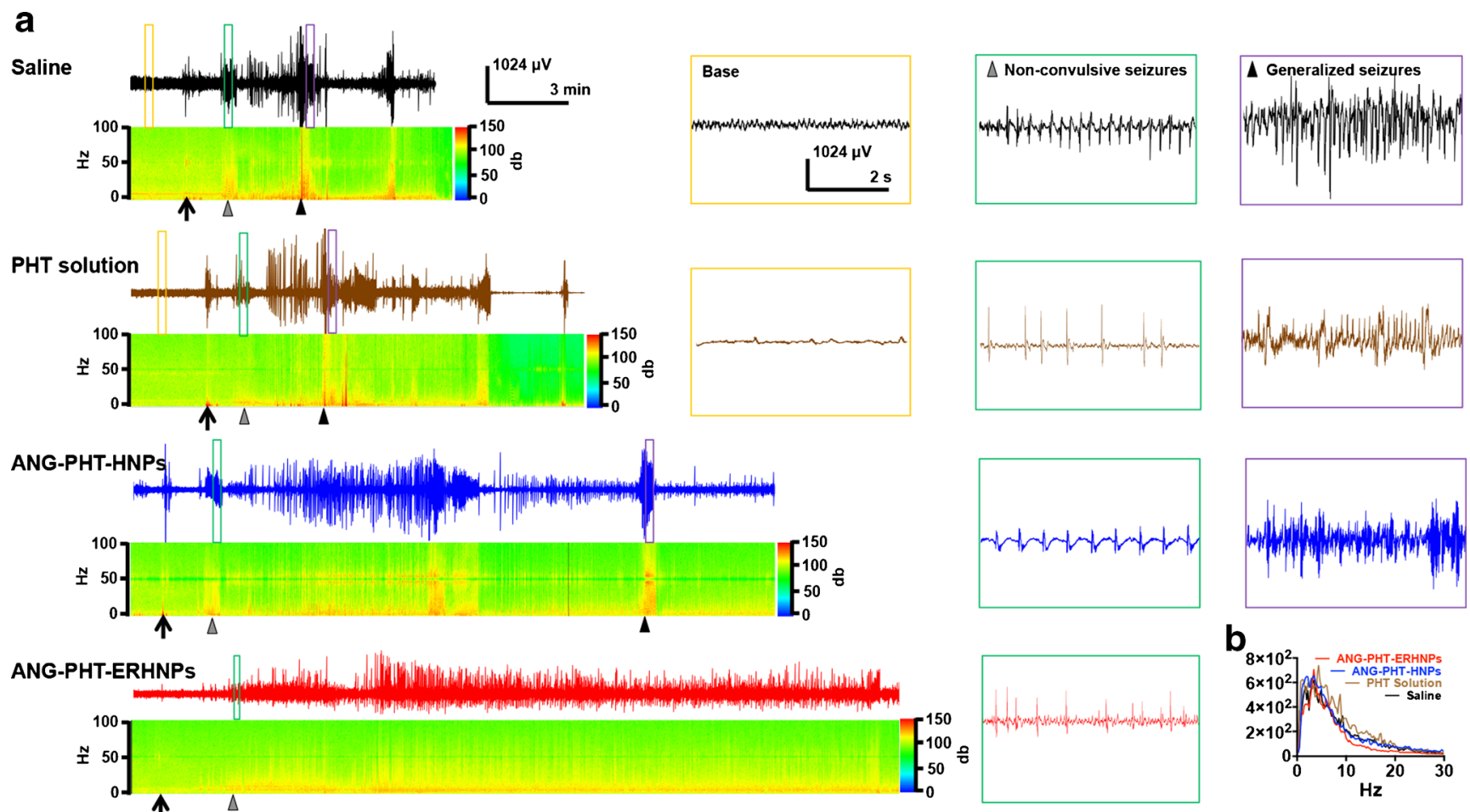

Fig. 4 Representative electroencephalographies (EEGs) during pentylenetetrazole (PTZ)-induced seizures. (a) Representative EEGs recorded from the hippocampus of rats and corresponding power spectral analysis during PTZ-induced seizures when rats were injected with saline, phenytoin sodium (PHT) solution, angiopep-2 (ANG)-PHT-hydrogel

slower elimination of PHT, making the area under the curve values greater than those of the PHT solution (Fig. 6C), suggesting the brain level of PHT was increased. Although PHT molecules were enveloped in the nanoparticles, treatment with ANG-PHT-HNP and ANG-PHT-ERHNP caused similar extracellular free levels of PHT as with treatment with PHT solution, which might result from the higher amount of total PHT in the CNS caused by brain-targeting characteristics of ANG. Importantly, in rats with seizure onset, the extracellular levels of PHT increased dramatically in ANG-PHT-ERHNP group but not in PHT solution group or ANG-PHT-HNP group (Fig. 6D-F). In addition, we found that the maximal PHT plasma level was similar with ANG-PHT-ERHNP and PHT solution treatment (Supplementary Fig. S2 and Supplementary Table S1), indicating that ANG-PHTERHNP treatment may improve the brain/plasma ratio of PHT.

To further evaluate whether seizure-triggered PHT release is correlated with the seizure intensity, we used different doses of PTZ to induce seizures of differing severity (Fig. 7A). The extracellular PHT levels from ANG-PHTERHNPs were much higher in mice dosed at $80 \mathrm{mg} / \mathrm{kg}$ than in mice dosed at $40 \mathrm{mg} / \mathrm{kg}$ (Fig. 7B, C), indicating that the levels of released PHT correlated with the severity of epileptic seizure onset. nanoparticles (HNPs) or ANG-PHT-electroresponsive HNPs (ERHNPs) $(20 \mathrm{mg} / \mathrm{kg})$; arrow, PTZ injection time; grey triangle, the initial time of nonconvulsive seizure onset; black triangle, the initial time of generalized seizure onset; right panel, enlarged views of boxes in the EEGs. (b) Spectrum analysis of the EEG

\section{Discussion}

Stimuli-responsive "smart" nanoparticles that respond to heat, $\mathrm{pH}$, enzyme activity, or magnetic fields have recently attracted great interest in biomedical research [23-27]. Few studies have explored the triggered release of nanoparticles mediated by an electric field $[28,29]$. In this study, we developed electroresponsive nanoparticles and demonstrated that the triggered-release of PHT from our system was sensitive to an electric field in vitro (as fast as $1 \mathrm{~min}$ ). Based on the abnormal EEG activity during seizure onset, we further successfully applied this system to in vivo pathological seizure models. Using microdialysis, we showed that ANG-PHTERHNP released large amounts of the nonprotein-bound form of PHT during seizures. Those results demonstrated that an extracellular field potential change during the onset of a seizure onset is enough to trigger the release of PHT, indicating its practicality for clinical prophylactically control of seizure in the future.

The electroresponsive effect was attributed to the electrical networks of NaSS in the hydrogel nanoparticles, as the HNPs (formula 1) was insensitive to the electric field, both in vitro and in vivo; these did not contain NaSS. Further, we found that the higher concentration of NaSS, the larger fold increased in the diameter of nanoparticles under electrical field, indicating 
Fig. 5 The in vivo antiepileptic effect of the nanoparticles in pilocarpine model. (a) Design of experiment; (b-e) the effect of nanoparticles at different doses on seizures stage (d), incidence of death (e), latency to generalized seizure (GS) (f), and latency to status epilepticus (SE) (g); the number of rats used in the experiment is indicated in the column of $\mathrm{B}\left({ }^{*} p<0.05\right.$, ${ }^{* *} p<0.01$ compared with first group; ${ }^{\#} p<0.05,{ }^{\# \#} p<0.01$ compared with groups with same dose; $\chi^{2}$ tests were used to compare the incidence of death; Kruskal-Wallis ANOVA followed by post-hoc Dunn's tests were used to compare seizure stage; and 1-way ANOVA followed by a post-hoc fishers least significant difference (LSD) test were used to compare latency to GS and SE $\mathrm{PHT}=$ phenytoin sodium; $\mathrm{ANG}=$ angiopep-2; $\mathrm{HNP}=$ hydrogel nanoparticles; ERHNP = electroresponsive HNP; EEG = electroencephalography
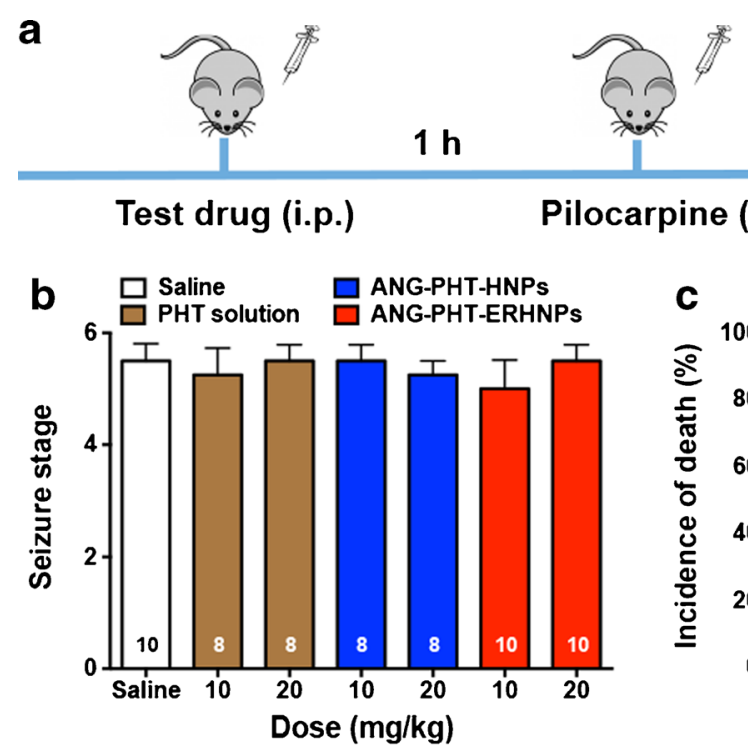

Pilocarpine (i.p.)

$60 \mathrm{~min}$
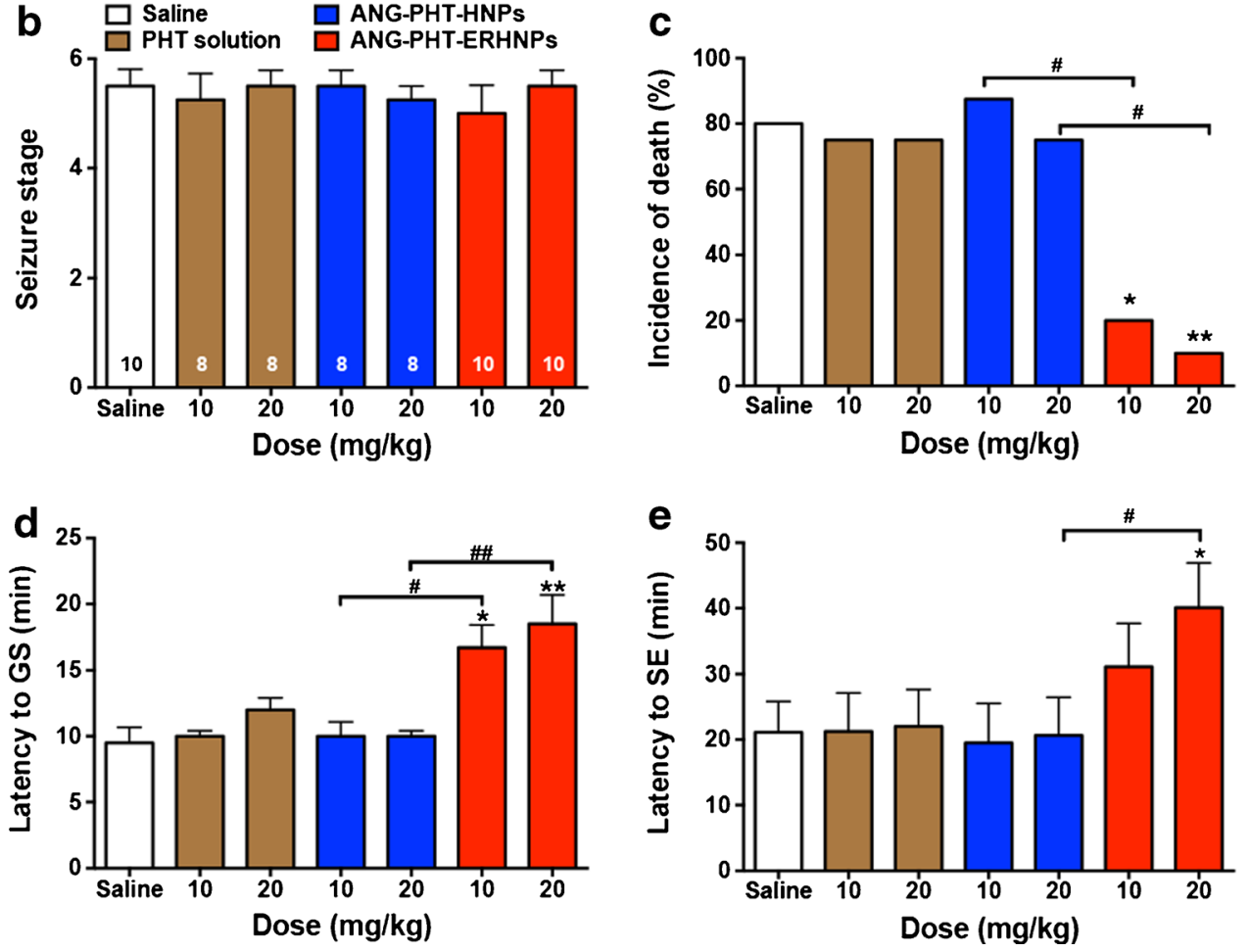

that the electrical field-responsive ability of nanoparticle is positively correlated with the NaSS content. The conductivity of the hydrogel nanoparticles induced by NaSS was reported to accelerate the ionization of the sulfo groups in the polymer chains. The increased degree of ionization of the polyelectrolyte hydrogel under an electric field are responsible for the higher swelling ratio, owing to increased electrostatic repulsion and hydrophilicity in the polymer chains [30], which transform electrical energy into mechanical energy and thus release the drug. The mechanism of PHT released from ANGPHT-ERHNP may be attributed to the effects of ANG-PHTERHNP swelling combined with PHT diffusion. In pH 7.4 PBS, the rates of ANG-PHT-ERHNP swelling and PHT diffusion from ANG-PHT-ERHNP were slow, thus a relatively slow PHT release was observed. Under an external electric field, the electrostatic repulsion of polyelectrolyte ionic (sulfonate anion) increased, hydrogel nanoparticles swelled faster, then the particle size of the ANG-ERHNP increased and the network structure of hydrogel nanoparticles loosed, so PHT released from the ANG-PHT-ERHNP increased greatly. However, the concentration of NaSS in preparation formula cannot be too high, because NaSS content is also negatively correlated with the original particle size, which is an important factor to influence the amount of drug loading. Thus, reasonable proportion of NaSS in formula is important for the electroresponsive behavior of nanoparticles.

Importantly, in all seizure models, ANG-PHT-ERHNP were able to produce antiseizure effects, even at a dose of 10 or $20 \mathrm{mg} / \mathrm{kg}$, compared with ANG-PHT-HNP, indicating that it lowered the effective therapeutic dose of PHT. This improved antiepileptic effect was due to the largely increased free (nonprotein-bound) levels of PHT released from ANGPHT-ERHNP, which was triggered by the seizure activities. Thus, it is feasible that an extracellular field potential change during the onset of a seizure is enough to trigger the release of PHT. Moreover, the levels of released PHT correlated with the severity of epileptic seizure onset, suggesting that electroresponsive ability was sensitive to the change of field potential. Those results indicated that these nanoparticles could quickly release the AEDs and produce an antiseizure effect, once the onset of epileptic seizures occurs. However, the ANG-PHT-ERHNP did not lengthen the latency of nonconvulsive seizures. The reasons may be that 1) PHT itself only protects against generalized tonic-clonic seizures but not the nonconvulsive seizures [3, 31]; 2) released PHT triggered by seizure activity cannot prevent the occurrence of seizures 
a

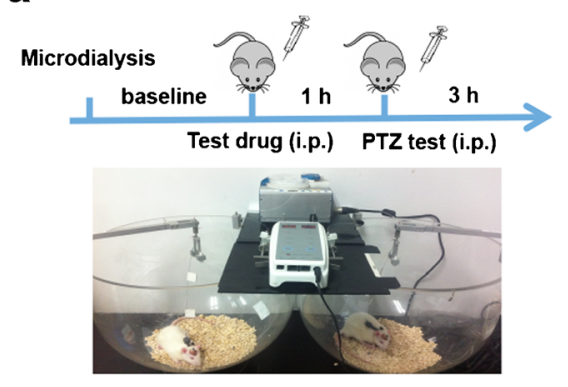

d

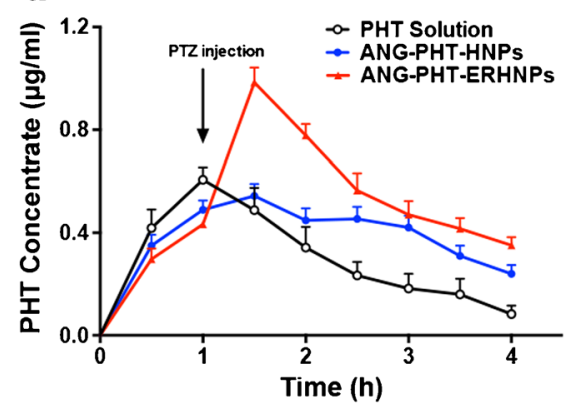

b

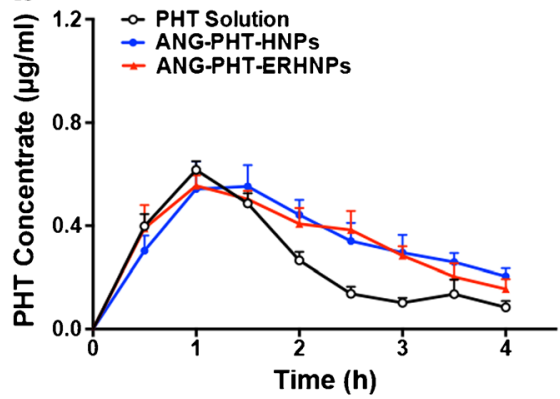

e

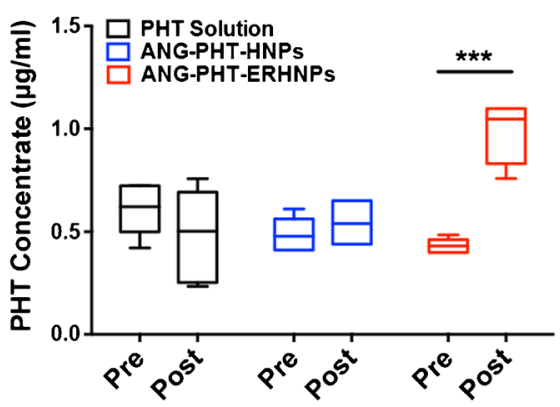

C

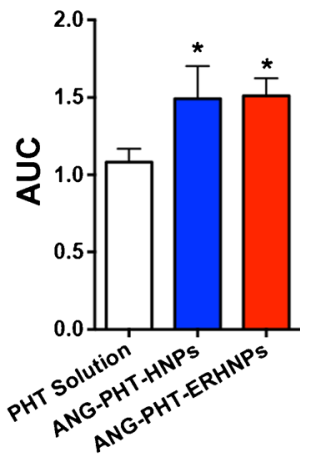

f

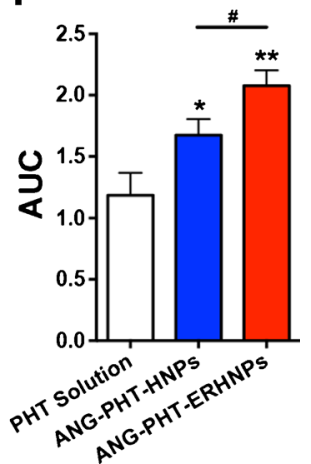

Fig. 6 The in vivo electroresponsive release of phenytoin sodium (PHT) from nanoparticles during seizures. (a) Design of the microdialysis experiment ( $n=6$ for each group); (b) PHT time profiles in brain dialysate of naïve rat after an intraperitoneal injection of PHT at a dose of $20 \mathrm{mg} / \mathrm{kg}$; (c) statistics of area under the curve (AUC) in (b) $\left({ }^{*} p<0.05\right.$, compared with the first group; 1-way ANOVA followed by post-hoc LSD tests); (d) PHT time profiles in brain dialysate of epileptic rat after an intraperitoneal injection of PHT at a dose of $20 \mathrm{mg} / \mathrm{kg}$; (e) free PHT concentration in brain dialysate before and $0.5 \mathrm{~h}$ after PTZ-induced seizures; $\left(* * * p<0.001\right.$, paired $t$-test); (f) statistics of AUC in (d) $\left({ }^{*} p<0.05\right.$, $* * p<0.01$ compared with first group, 1-way ANOVA followed by post-hoc fishers least significant difference (LSD) tests) PTZ = pentylenetetrazole; $\mathrm{ANG}=$ angiopep-2; HNP = hydrogel nanoparticles; $\mathrm{ERHNP}=$ electroresponsive HNP; EEG = electroencephalography but might rather affect spread of seizure activity along with an impact on seizure severity. At present, closed-loop neurostimulation is a new alternative treatment for epilepsy [32-34], which means that neurostimulation was applied to control seizure onset immediately upon a seizure being detected by an automatic EEG algorithm [35]. However, it is an invasive intervention, and the implantable system for seizure detection still costs a great deal. It is conceivable that our electroresponsive nanoparticles may be suitable for clinical on-demand control of epilepsy in the future, in a way similar to closed-loop neurostimulation, with the advantages of convenience and relatively lower cost. a

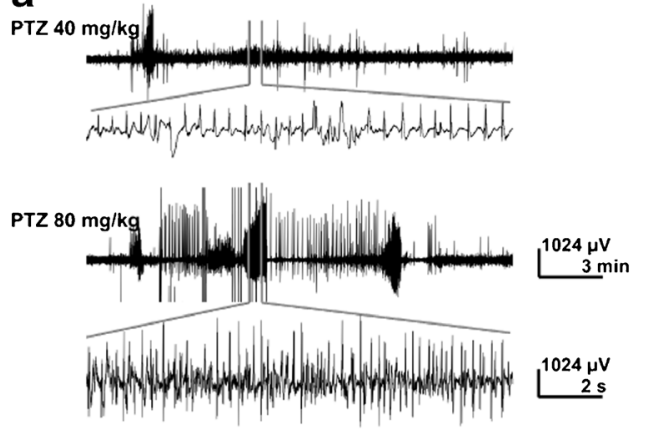

Fig. 7 The in vivo electroresponsive release of phenytoin sodium PHT from nanoparticles during seizures with different intensity. (a) Representative electroencephalographies (EEGs) recorded from the hippocampus of rats during seizures induced by pentylenetetrazole b

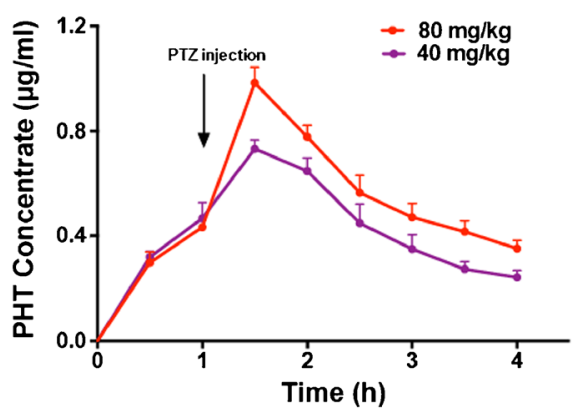

C

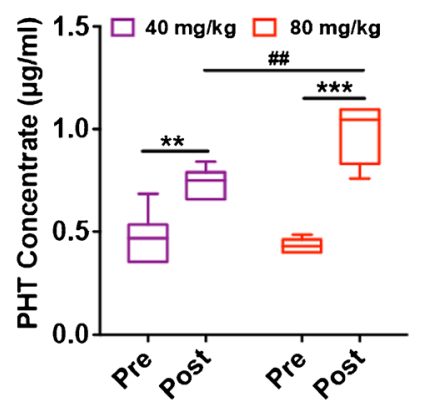

(PTZ) at different doses; (b) PHT time profiles in brain dialysate of epileptic rats with different seizure intensity; (c) free PHT concentration in brain dialysate before and $0.5 \mathrm{~h}$ after seizures with different intensity $\left({ }^{* *} p<0.01, * * * p<0.001\right.$, paired $t$-test, ${ }^{\# \#} p<0.01 t$-test $)$ 
Despite advances in pharmacotherapy for epilepsy, $>30 \%$ of patients still have incompletely controlled seizures [36]. One of the main reasons is that the expression or function of multidrug transporters in the brain is augmented in epilepsy, leading to impaired access to the targets of CNS for AEDs [37, 38]. The relatively narrow therapeutic window of AEDs results in intolerable side effects when one tries to increase dosages. In the present study, although the total PHT plasma level was similar with ANG-PHT-ERHNP and PHT solution treatment, the brain level of PHT in ANG-PHT-ERHNP was largely increased compared with PHT solution, indicating that ANGPHT-ERHNP treatment improved the brain/plasma ratio of PHT. This effect may be due to the brain targeting effect of ANG [39], and/or nanoparticle characteristics such as small size and prolonged blood circulation owing to PEGylation [40]. In addition, our previous study in a kindling model further verified that the antiseizure effects of the ANG-PHT-ERHNP could last as long as $8 \mathrm{~h}$; the effect

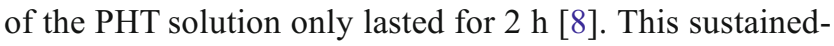
release characteristic of the carrier can effectively reduce the number of times the medicine needs to be administered. All these advantages of ANG-ERHNP suggest promising clinical prospects. In the present study, we used PHT because 1) PHT remains one of the most widely used AED drugs in the world, although it has now been relegated to second-line therapy status in Europe and America owing to the discovery of its nonlinear kinetics properties, extensive interactions, and potential side effects [41];2) the current formula of ANG-ERHNP is only suitable for lipophilic drugs; 3 ) the amount of drug loading for ANG-ERHNP is limited. Thus, further study will be required to optimize or update the formulae for better permeability across the BBB of other hydrophilic AEDs that do not easily enter the brain, such as valproic acid.

In conclusion, here we provide in vivo evidence that ANGPHT-ERHNP have electroresponsive ability, which is determined by the content of NaSS in the preparation formula. Once epileptiform activities occur, PHT will be released from ANG-PHT-ERHNP and exert antiseizure effect in generalized tonic-clonic seizure models. These electroresponsive nanoparticles may update the therapeutic paradigm of existing drug treatment for epilepsy.

Acknowledgments This project was supported by grants from the National Natural Science Foundation of China (91332202, 81202478, 81273492 and 81471316 ) and the Program for Zhejiang Leading Team of S\&T Innovation (2011R50014).

\section{Compliance with Ethical Standards}

Required Author Forms Disclosure forms provided by the authors are available with the online version of this article.

\section{References}

1. Rao VR, Lowenstein DH. Epilepsy. Curr Biol 2015;25:R742R746.

2. Bialer M, White HS. Key factors in the discovery and development of new antiepileptic drugs. Nat Rev Drug Discov 2010;9:68-82.

3. Shorvon SD. Drug treatment of epilepsy in the century of the ILAE: the first 50 years, 1909-1958. Epilepsia 2009;50(Suppl. 3):69-92.

4. Glauser T, Ben-Menachem E, Bourgeois B, et al. Updated ILAE evidence review of antiepileptic drug efficacy and effectiveness as initial monotherapy for epileptic seizures and syndromes. Epilepsia 2013;54:551-563.

5. Bennewitz MF, Saltzman WM. Nanotechnology for delivery of drugs to the brain for epilepsy. Neurotherapeutics 2009;6:323-336.

6. Liu J, He Y, Zhang J, et al. Functionalized nanocarrier combined seizure-specific vector with P-glycoprotein modulation property for antiepileptic drug delivery. Biomaterials 2015;74:64-76.

7. Xu L, Zhang H, Wu Y. Dendrimer advances for the central nervous system delivery of therapeutics. ACS 2014;5:2-13.

8. Ying X, Wang Y, Liang J, et al. Angiopep-conjugated electro-responsive hydrogel nanoparticles: therapeutic potential for epilepsy. Angew Chem Int Ed Engl 2014;53:12436-12440.

9. McNamara JO, Rigsbee LC, Butler LS, Shin C. Intravenous phenytoin is an effective anticonvulsant in the kindling model. Ann Neurol 1989;26:675-678.

10. Du YZ, Tomohiro T, Zhang G, Nakamura K, Kodaka M. Biotinylated and enzyme-immobilized carrier prepared by heterobifunctional latex beads. Chem Commun 2004:616-617.

11. Wu DC, Zhu-Ge ZB, Yu CY, et al. Low-frequency stimulation of the tuberomammillary nucleus facilitates electrical amygdaloidkindling acquisition in Sprague-Dawley rats. Neurobiol Dis 2008;32:151-156.

12. Paxinos G, Watson CR, Emson PC. AChE-stained horizontal sections of the rat brain in stereotaxic coordinates. J Neurosci Methods 1980;3:129-149.

13. Zhang SH, Sun HL, Fang Q, et al. Low-frequency stimulation of the hippocampal CA3 subfield is anti-epileptogenic and anti-ictogenic in rat amygdaloid kindling model of epilepsy. Neurosci Lett 2009;455:51-55.

14. Zhong $\mathrm{K}, \mathrm{Wu} \mathrm{DC}$, Jin MM, et al. Wide therapeutic time-window of low-frequency stimulation at the subiculum for temporal lobe epilepsy treatment in rats. Neurobiol Dis 2012;48:20-26.

15. Xu Z, Wang Y, Jin M, et al. Polarity-dependent effect of lowfrequency stimulation on amygdaloid kindling in rats. Brain Stimul 2013;6:190-197.

16. Wang $\mathrm{Y}, \mathrm{Xu} \mathrm{Z}$, Cheng $\mathrm{H}$, et al. Low-frequency stimulation inhibits epileptogenesis by modulating the early network of the limbic system as evaluated in amygdala kindling model. Brain Struct Funct 2014;219:1685-1696.

17. Racine RJ. Modification of seizure activity by electrical stimulation. II. Motor seizure. Electroencephalogr Clin Neurophysiol 1972;32: 281-294.

18. Engel J, Jr., Wolfson L, Brown L. Anatomical correlates of electrical and behavioral events related to amygdaloid kindling. Ann Neurol 1978;3:538-544.

19. Loscher W. Critical review of current animal models of seizures and epilepsy used in the discovery and development of new antiepileptic drugs. Seizure 2011;20:359-368.

20. Morrisett RA, Jope RS, Snead OC, 3rd. Effects of drugs on the initiation and maintenance of status epilepticus induced by administration of pilocarpine to lithium-pretreated rats. Exp Neurol 1987;97:193-200.

21. Blanco MM, dos Santos JG, Jr., Perez-Mendes P, et al. Assessment of seizure susceptibility in pilocarpine epileptic and nonepileptic 
Wistar rats and of seizure reinduction with pentylenetetrazole and electroshock models. Epilepsia 2009;50:824-831.

22. Walker MC, Alavijeh MS, Shorvon SD, Patsalos PN. Microdialysis study of the neuropharmacokinetics of phenytoin in rat hippocampus and frontal cortex. Epilepsia 1996;37:421-427.

23. Mura S, Nicolas J, Couvreur P. Stimuli-responsive nanocarriers for drug delivery. Nat Mater 2013;12:991-1003.

24. Chen KJ, Liang HF, Chen HL, et al. A thermoresponsive bubblegenerating liposomal system for triggering localized extracellular drug delivery. ACS Nano 2013;7:438-446.

25. Du JZ, Du XJ, Mao CQ, Wang J. Tailor-made dual $\mathrm{pH}$-sensitive polymer-doxorubicin nanoparticles for efficient anticancer drug delivery. J Am Chem Soc 2011;133:17560-17563.

26. Bernardos A, Mondragon L, Aznar E, et al. Enzyme-responsive intracellular controlled release using nanometric silica mesoporous supports capped with "saccharides". ACS Nano 2010;4:63536368.

27. Zhang F, Braun GB, Pallaoro A, et al. Mesoporous multifunctional upconversion luminescent and magnetic "nanorattle" materials for targeted chemotherapy. Nano Lett 2012;12:61-67.

28. Yan Q, Yuan J, Cai Z, Xin Y, Kang Y, Yin Y. Voltage-responsive vesicles based on orthogonal assembly of two homopolymers. J Am Chem Soc 2010;132:9268-9270.

29. Ge J, Neofytou E, Cahill TJ, 3rd, Beygui RE, Zare RN. Drug release from electric-field-responsive nanoparticles. ACS Nano 2012;6:227-233.

30. Lim HS, Lee JH, Walish JJ, Thomas EL. Dynamic swelling of tunable full-color block copolymer photonic gels via counterion exchange. ACS Nano 2012;6:8933-8939.
31. Rogawski MA, Loscher W. The neurobiology of antiepileptic drugs. Nat Rev Neurosci 2004;5:553-564.

32. Theodore WH, Fisher RS. Brain stimulation for epilepsy. Lancet Neurol 2004;3:111-118.

33. $\mathrm{Xu} \mathrm{ZH,} \mathrm{Wu} \mathrm{DC,} \mathrm{Fang} \mathrm{Q,} \mathrm{et} \mathrm{al.} \mathrm{Therapeutic} \mathrm{time} \mathrm{window} \mathrm{of} \mathrm{low-}$ frequency stimulation at entorhinal cortex for amygdaloid-kindling seizures in rats. Epilepsia 2010;51:1861-1864.

34. Berenyi A, Belluscio M, Mao D, Buzsaki G. Closed-loop control of epilepsy by transcranial electrical stimulation. Science 2012;337: 735-737.

35. Vonck K, Boon P. Epilepsy: closing the loop for patients with epilepsy. Nat Rev Neurol 2015;11:252-254.

36. Loscher W, Klitgaard H, Twyman RE, Schmidt D. New avenues for anti-epileptic drug discovery and development. Nat Rev Drug Discov 2013;12:757-776.

37. Szoeke CE, Newton M, Wood JM, et al. Update on pharmacogenetics in epilepsy: a brief review. Lancet Neurol 2006;5:189-196.

38. Remy S, Beck H. Molecular and cellular mechanisms of pharmacoresistance in epilepsy. Brain 2006;129:18-35.

39. Xin H, Sha X, Jiang X, et al. The brain targeting mechanism of Angiopep-conjugated poly(ethylene glycol)-co-poly(epsiloncaprolactone) nanoparticles. Biomaterials 2012;33:1673-1681.

40. Frigell J, Garcia I, Gomez-Vallejo V, Llop J, Penades S. 68Galabeled gold glyconanoparticles for exploring blood-brain barrier permeability: preparation, biodistribution studies, and improved brain uptake via neuropeptide conjugation. J Am Chem Soc 2014;136:449-457.

41. Shorvon SD. Drug treatment of epilepsy in the century of the ILAE: the second 50 years, 1959-2009. Epilepsia 2009;50(Suppl. 3):93130. 